\title{
Value of combined perfusion and metabolic tracers versus stress-rest perfusion imaging for coronary heart disease
}

\author{
Kenichi Nakajima, MD, PhD, ${ }^{a}$ and Hiroshi Wakabayashi, MD, PhD ${ }^{b}$ \\ a Department of Functional Imaging and Artificial Intelligence, Kanazawa University Graduate \\ School of Medicine, Kanazawa, Japan \\ b Department of Nuclear Medicine, Kanazawa University Hospital, Kanazawa, Japan
}

Received May 15, 2020; accepted May 15, 2020

doi: $10.1007 / \mathrm{s} 12350-020-02206-0$

See related article, pp. 3070-3080

\section{IS THE IDIOMATIC PROVERB, TWO HEADS ARE BETTER THAN ONE, TRUE FOR RADIOTRACERS?}

Stress-rest myocardial perfusion imaging (MPI) has become the standard procedure for assessing coronary artery disease (CAD), and it is also used in clinical practice to evaluate stress-induced ischemia and infarction. Stress studies are the standard approach to CAD according to clinical practice guidelines in the USA and Europe, ${ }^{1}$ as well as in Japan. ${ }^{2}$ One of the greatest advantages of nuclear cardiology is the ability to assess perfusion flow reserve in response to stress imposed by exercise or pharmacologically. However, flow reserve, whether estimated relatively or absolutely, cannot be determined only from a baseline resting state.

The first-line indication for the stress method is exercise, which is the most prevalent type of physiological stress as it reflects daily activities. However, when patients are unable to exercise, pharmacological stress using a coronary vasodilator such as adenosine or an $\mathrm{A}_{2 \mathrm{~A}}$ adenosine receptor agonist such as regadenoson is indicated. Most patients even those with multivessel disease can tolerate a pharmacological stress study if appropriate care is taken during the procedure. Low-

Reprint requests: Kenichi Nakajima, MD, PhD, Department of Functional Imaging and Artificial Intelligence, Kanazawa University Graduate School of Medicine, 13-1 Takaramachi, Kanazawa9208640, Japan; nakajima@med.kanazawa-u.ac.jp

J Nucl Cardiol 2021;28:3081-4.

$1071-3581 / \$ 34.00$

Copyright (C) 2020 American Society of Nuclear Cardiology. level treadmill or ergometer exercise during adenosine infusion is safe and helps to reduce side effects such as hypotension.

Nuclear cardiology is still underutilized in Japan considering the value of MPI with single-photon emission computed tomography (SPECT). The Japanese Registry of all Cardiac and Vascular Diseases (JROAD) report issued by the Japanese Circulation Society describes 225,000 MPI studies that were undertaken during $2017 .{ }^{3}$ In addition, approximately 63,500 cardiac studies were conducted with ${ }^{123}$ I-betamethyliodophenyl pentadecanoic acid (BMIPP) and ${ }^{123}$ I-meta-iodobenzylguanidine $(m \mathrm{IBG})$ during $2017 .{ }^{4}$ The Japanese Ministry of Health, Labor, and Welfare revised the indications eligible for reimbursement of the medical costs of percutaneous coronary intervention during 2018 to include stenosis of $\geq 90 \%$ of the coronary artery and/ or functional ischemia determined using an appropriate diagnostic method. ${ }^{5}$ Therefore, proven ischemia has become an essential requirement for subsequent coronary intervention, emphasizing ischemia-guided treatment.

In this context, the following question may arise: Is combined use of two tracers (namely perfusion and metabolism) better than one perfusion tracer for the diagnosis of CAD?

\section{POSITRON EMISSION TOMOGRAPHY (PET) VS SPECT}

High-resolution images, the short half-life of positron tracers, absolute quantitation of myocardial blood flow, and hence better diagnostic accuracy, are advantages offered by PET compared with SPECT. However, its application still depends on local healthcare systems and reimbursement policies. Although both ${ }^{18} \mathrm{~F}$-fluorodeoxyglucose (FDG) and ${ }^{13} \mathrm{~N}$-ammonia $\left(\mathrm{NH}_{3}\right)$ PET 
have been approved for clinical use in Japan, their application to patients with CAD remains limited. However, since the detection of ischemia and the viability of myocardial regions at risk is essential for choosing subsequent treatment strategies such as coronary revascularization, the roles of PET tracers are likely to expand. The important advantage of PET is the ability to quantify absolute blood flow in units of $\mathrm{mL} / \mathrm{min} / \mathrm{g}$, and myocardial flow reserve in response to pharmacological stress. High-resolution and high sensitivity combined with scatter and attenuation correction have enabled the performance of PET to exceed that of SPECT.

Technological advances in current SPECT systems have been directed towards absolute quantitation using cadmium zinc telluride and Anger cameras. While PET remains the better method of quantitation, SPECT might be able to catch up in the near future for screening patients because it can measure absolute flow reserve. Developments in dynamic acquisition, data processing, modeling, and new tracers with better extraction properties than ${ }^{99 \mathrm{~m}}$ Tc-methoxyisobutylisonitrile (MIBI) and tetrofosmin are also important steps for such progress. ${ }^{6,7}$

\section{${ }^{18}$ F-FDG AND REST PERFUSION}

In this issue of Journal of Nuclear Cardiology, Wang and colleagues reported an evaluation of the ability of ${ }^{13} \mathrm{~N}-\mathrm{NH}_{3}$ perfusion at rest combined with ${ }^{18} \mathrm{~F}$ FDG metabolic imaging to detect coronary stenosis in CAD. ${ }^{8}$ Machine learning improved the diagnostic accuracy of the combined approach over resting myocardial perfusion PET alone, particularly when a mismatch between the tracers was included. The authors suggested that ${ }^{13} \mathrm{~N}-\mathrm{NH}_{3}$ perfusion and ${ }^{18} \mathrm{~F}$-FDG could serve as a gatekeeper for coronary angiography under the Chinese healthcare system.

Dual-isotope simultaneous acquisition using SPECT cameras was proposed in the 1990s to simultaneously evaluate resting cardiac perfusion and metabolism with a combination of ${ }^{99 \mathrm{~m}} \mathrm{Tc}-\mathrm{MIBI} /{ }^{18} \mathrm{~F}$ FDG and an ultra-high-energy collimator. ${ }^{9}$ That strategy resulted in $100 \%$ sensitivity with a positive predictive value of $93 \%$, thus showing its potential for diagnosing CAD when $>70 \%$ stenosis was a criterion.

However, the Japanese healthcare system does not permit the routine clinical use of ${ }^{18} \mathrm{~F}-\mathrm{FDG}$ and ${ }^{13} \mathrm{~N}-\mathrm{NH}_{3}$ to detect ischemia. ${ }^{5}$ From a pathophysiological viewpoint, occult ischemia overlooked by resting perfusion could be visualized when a mismatch is added. An ischemic region is likely to appear as a mismatch between perfusion and metabolism, and an infarct region will appear as perfusion/metabolism matched defects. Our clinical experience with fatty acid imaging using
${ }^{123}$ I-BMIPP, which was approved in Japan in 1993, supports this notion. ${ }^{10}$

\section{PERFUSION AND FATTY ACID METABOLISM}

The clinical guidelines for stable CAD published by the Japanese Circulation Society summarizes the indications and recommendations for ${ }^{123}$ I-BMIPP imaging. 2,10 Since myocardial "ischemic memory", identified at rest is the basic concept for applying fatty acid imaging to ischemia, other pathological states, such as myocardial ischemia, coronary spastic angina, ${ }^{11}$ hibernating myocardium, ${ }^{12}$ and the identification of myocardial ischemia or injury in hemodialysis ${ }^{13}$, are included. The guidelines further list the prognosis of patients on hemodialysis and other cardiac diseases as indications of ${ }^{123}$ I-BMIPP imaging. ${ }^{14}$

Metabolic damage to fatty acids is generally considered to depend on the severity of ischemia. The diagnostic accuracy is higher in acute coronary syndrome and more severe conditions, and lower in chronic stable conditions or less severe conditions. Thus, as shown by Wang et al. ${ }^{8}$, the enhanced ability of to detect perfusion and glucose metabolism compared with resting perfusion alone might be understandable, because mismatches between perfusion and metabolism are more frequent when patients have multivessel diseases and complications of diabetes.

\section{COMPARISON OF STRESS AND REST FLOW, FATTY ACID, AND GLUCOSE UPTAKE}

Even in chronic stable CAD, more frequently induced, recent, or persistent ischemia including asymptomatic ischemia might result in a regional or local mismatch between fatty acid uptake and perfusion. This mismatch is sometimes understood as, " ${ }^{123} \mathrm{I}$-BMIPP memory imaging.' Findings of ${ }^{123}$ I-BMIPP imaging might also become positive even when stress MPI is negative such as in multivessel disease, exercise insufficiency, and complications of heart failure. Moreover, multiple BMIPP defects might be derived from multivessel disease and microvascular or cellular derangement.

The potential of ${ }^{18} \mathrm{~F}$-FDG for imaging ischemia has been assessed in relatively small studies, which consistently revealed increased FDG uptake in regions with reversible perfusion abnormalities. ${ }^{15,16}$ Whether such " direct ischemia imaging", can serve as an alternative to stress-rest perfusion, and which pathophysiological states are suitable for combined FDG/perfusion imaging need to be investigated, for example, in patients with multivessel disease and diabetes mellitus. 
Regardless, from a simplified viewpoint, a dissociation between fatty acid/perfusion and FDG/perfusion is a complementary aspect of ischemia that reflects a local metabolic shift from reduced fatty acid, to increased glucose metabolic activities. Similar considerations might apply to viability assessment.

\section{MACHINE LEARNING VS STATISTICAL ANALYSIS}

Another aspect to be considered is the application of machine learning to the data analysis, diagnosis, and prognosis of cardiovascular diseases. ${ }^{17}$ Wang et al. ${ }^{8}$ selected myocardial blood flow at rest, scar from ${ }^{18} \mathrm{~F}$ FDG, and a mismatch between perfusion and ${ }^{18}$ F-FDG as variables that could predict coronary stenosis of $\geq 75 \%$. The diagnostic accuracy did not significantly differ between rest perfusion and the machine learning approach when stenosis was $90 \%$ and $50 \%$, suggesting that machine learning was effective under specific conditions. This could have been partly due to the small patient cohort $(n=88)$ used for training, so data from a much larger patient cohort should be consistently implemented to improve diagnostic accuracy. Since the number of variables was relatively small, a conventional univariable and multivariable models might have been used for analysis. However, the purpose of their study was not to compare statistical and machine learning models; to this aspect, it was not investigated. ${ }^{8}$ Since a support vector machine using only a few variables provided better diagnostic accuracy than classical statistical methods, a machine learning-based classifier might become a novel method of choice. In addition, more complicated clinical factors such as symptoms, electrocardiographic changes, and laboratory data could be included in training machine learning. Instead of using coronary stenosis as the truth for training machine learning, the results of studies of ischemia with stress-rest perfusion, fractional flow reserve, and prognosis after coronary intervention might also serve as gold standards.

We applied machine learning to predict sudden cardiac death and end-stage heart failure in 526 patients with chronic heart failure using 13 clinical variables including ${ }^{123}$ I- $m$ IBG indices. ${ }^{18}$ Since the differential prediction of death modes was not achieved by conventional statistical models, ${ }^{19}$ the application of artificial intelligence seemed to represent a viable option for analyzing more complicated interactions among categorical and continuous variables, as well as various cardiovascular imaging modalities. ${ }^{17}$

Machine learning will become applied more extensively to clinical research investigations that have historically used classical statistical models. Whether classifiers and predictors created by machine learning would be applicable to different clinical settings requires further evaluation, and training and test sets should be optimized to create reliable models. However, a new approach using artificial intelligence might deliver new insights, even when meaningful results from $p$ values of individual variables cannot be extracted. Since diagnostic imaging is becoming more complex, decision support systems based on machine learning might provide greater automation and objectivity by integrating images and clinical background information in the near future. ${ }^{20}$

\section{METABOLIC-PERFUSION MISMATCH OR STRESS-REST}

Radiopharmaceutical or PET tracers such as ${ }^{18} \mathrm{~F}$ FDG, fatty acid imaging, and innervation imaging can visualize myocardial regions exposed to ischemic injury and might be applied even when exercise or pharmacological stress is not indicated. These applications are safe for patients who are frail or elderly, or those with severe disease status or heart failure.

There will be no doubt that stress-rest perfusion studies comprise the first line for the diagnosis and follow-up of CAD. However, instead of repeatedly assessing the same patients using stress-rest perfusion during the clinical course, appropriate use of metabolic imaging depending on patient status is certainly safe and practical for identifying potential ischemia, ischemic memory, and myocardial injury. Further comparative studies and more clinical evidence are required before proceeding to imaging modalities beyond perfusion.

\section{Acknowledgments}

The authors appreciate Norma Foster for editorial assistance.

\section{Disclosure}

$K$ Nakajima has collaborative researches with Nihon Medi Physics, Tokyo, Japan (supplier of $\left.{ }^{123} I-B M I P P\right)$, and Fujifilm Toyama Chemical, Tokyo, Japan (supplier of ${ }^{123} \mathrm{I}$ $m I B G)$. H. Wakabayashi has no conflicts of interest to declare.

\section{References}

1. Henzlova MJ, Duvall WL, Einstein AJ, Travin MI, Verberne HJ. ASNC imaging guidelines for SPECT nuclear cardiology procedures: Stress, protocols, and tracers. J Nucl Cardiol 2016;23:60639.

2. Japanese Circulation Society Working Group. JCS 2018 Guideline on Diagnosis of Chronic Coronary Heart Diseases (Revised 2018). 2019 [in Japanese]. (https://www.j-circ.or.jp/cms/wp-content/uplo ads/2020/02/JCS2018_yamagishi_tamaki.pdf). 
3. Japanese Circulation Society: Annual Report of The Japanese Registry Of All cardiac and vascular Diseases (JROAD) 2018 [in Japanese] http://www.j-circ.or.jp/jittai_chosa/jittai_chosa2017we b.pdf.

4. Subcommittee on Survey of Nuclear Medicine Practice in Japan: The Present State of Nuclear Medicine Practice in Japan: A Report of the 8th Nationwide Survey in 2017. RADIOISOTOPES. 2018 [In Japanese] (https://doi.org/10.3769/radioisotopes.67.339.

5. Yoshinaga K, Maruno H, Chikamori T. Updated Japanese Ministry of Health, Labour and Welfare Reimbursement Policy for cardiac positron emission tomography and coronary intervention. Ann Nucl Cardiol 2018. https://doi.org/10.17996/anc.18-00070.

6. Agostini D, Roule V, Nganoa C, Roth N, Baavour R, Parienti JJ, et al. First validation of myocardial flow reserve assessed by dynamic 99mTc-sestamibi CZT-SPECT camera: head to head comparison with 15O-water PET and fractional flow reserve in patients with suspected coronary artery disease. The WATERDAY study. Eur J Nucl Med Mol Imaging 2018;45:1079-90.

7. Giubbini R, Bertoli M, Durmo R, Bonacina M, Peli A, Faggiano I, et al. Comparison between $13 \mathrm{~N}-\mathrm{NH} 3-\mathrm{PET}$ and $99 \mathrm{mTc}$-Tetrofosmin-CZT SPECT in the evaluation of absolute myocardial blood flow and flow reserve. J Nucl Cardiol 2019. https://doi.org/10. 1007/s12350-019-01939-x.

8. Wang F, Xu W, Du D, Feng H, Zhang X, Wang S, et al. Evaluation of the diagnostic value of joint PET myocardial perfusion and metabolic imaging for vascular stenosis in patients with obstructive coronary artery disease. J Nucl Cardiol 2020. https://d oi.org/10.1007/s12350-020-02160-x.

9. Delbeke D, Videlefsky S, Patton JA, Campbell MG, Martin WH, Ohana I, et al. Rest myocardial perfusion/metabolism imaging using simultaneous dual-isotope acquisition SPECT with technetium-99m-MIBI/fluorine-18-FDG. J Nucl Med 1995;36:2110-9.

10. Matsumoto N, Hirayama A. Current Japanese Ministry of Health, Labor, and Welfare Approval of cardiac single photon emission computed tomography. Ann Nucl Cardiol 2015;1:108-9.

11. Nakajima K, Shimizu K, Taki J, Uetani Y, Konishi S, Tonami N, et al. Utility of iodine-123-BMIPP in the diagnosis and follow-up of vasospastic angina. J Nucl Med 1995;36:1934-40.
12. Hosokawa R, Nohara R, Fujibayashi Y, Hirai T, Fujita M, Magata $\mathrm{Y}$, et al. Myocardial metabolism of 123I-BMIPP during low-flow ischaemia in an experimental model: Comparison with myocardial blood flow and 18F-FDG. Eur J Nucl Med 2001;28:1630-9.

13. Moroi M, Tamaki N, Nishimura M, Haze K, Nishimura T, Kusano E, et al. Association between abnormal myocardial fatty acid metabolism and cardiac-derived death among patients undergoing hemodialysis: Results from a cohort study in Japan. Am J Kidney Dis 2013;61:466-75.

14. Inaba Y, Bergmann SR. Prognostic value of myocardial metabolic imaging with BMIPP in the spectrum of coronary artery disease: A systematic review. J Nucl Cardiol 2010;17:61-70.

15. Araujo LI, McFalls EO, Lammertsma AA, Jones T, Maseri A. Dipyridamole-induced increased glucose uptake in patients with single-vessel coronary artery disease assessed with PET. J Nucl Cardiol 2001;8:339-46.

16. Jain D, He ZX. Direct imaging of myocardial ischemia: A potential new paradigm in nuclear cardiovascular imaging. J Nucl Cardiol 2008;15:617-30.

17. Al'Aref SJ, Anchouche K, Singh G, Slomka PJ, Kolli KK, Kumar A, et al. Clinical applications of machine learning in cardiovascular disease and its relevance to cardiac imaging. Eur Heart $\mathbf{J}$ 2019;40:1975-86.

18. Nakajima K, Nakata T, Doi T, Tada H, Maruyama K. Machine learning-based risk model using 123I-metaiodobenzylguanidine to differentially predict modes of cardiac death in heart failure. $\mathbf{J}$ Nucl Cardiol 2020. https://doi.org/10.1007/s12350-020-02173-6.

19. Nakajima K, Nakata T, Doi T, Kadokami T, Matsuo S, Konno T, et al. Validation of 2-year 123I-meta-iodobenzylguanidine-based cardiac mortality risk model in chronic heart failure. Eur Heart $\mathbf{J}$ Cardiovasc Imagin 2018;19:749-56.

20. Garcia EV, Klein JL, Taylor AT. Clinical decision support systems in myocardial perfusion imaging. J Nucl Cardiol 2014;21:427-39.

Publisher's Note Springer Nature remains neutral with regard to jurisdictional claims in published maps and institutional affiliations. 